\title{
Bernard Arcand
}

Anthropologue, professeur au département d'anthropologie, Université Laval

(1979)

\section{"Production, culture et idéologie: approche structuraliste”}

Un document produit en version numérique par Jean-Marie Tremblay, bénévole, professeur de sociologie au Cégep de Chicoutimi

Courriel: jean-marie tremblay@uqac.ca

Site web pédagogique : http://www.uqac.ca/jmt-sociologue/

Dans le cadre de la collection: "Les classiques des sciences sociales"

Site web: http://www.uqac.ca/Classiques_des_sciences_sociales/

Une collection développée en collaboration avec la Bibliothèque

Paul-Émile-Boulet de l'Université du Québec à Chicoutimi

Site web: http://bibliotheque.uqac.ca/ 
Cette édition électronique a été réalisée par Jean-Marie Tremblay, bénévole, professeur de sociologie au Cégep de Chicoutimi à partir de l'article de :

Bernard Arcand

Anthropologue, professeur au département d’anthropologie, Université Laval.

“Production, culture et idéologie : approche structuraliste.”

Un article publié dans l'ouvrage collectif intitulé: Perspectives anthropologiques. Un collectif d'anthropologues québécois, chapitre 10, pp. 143 à 153.

Montréal: Les Éditions du Renouveau pédagogique, 1979, 436 pp.

[Autorisation formelle de diffuser ce texte accordée, le 13 novembre 2005, par l'auteur, et le 14 novembre 2005 par l'éditeur, Les Éditions du Renouveau pédagogique.]

\begin{tabular}{|l|} 
M. Bernard Arcand: Bernard.Arcand@ant.ulaval.ca \\
Mme Chantal Bordeleau, représentante de l’éditeur, \\
Les Éditions du Renouveau pédagogique à Montréal : \\
$\underline{\text { chantal.bordeleau@erpi.com }}$
\end{tabular}

Polices de caractères utilisée :

Pour le texte: Times New Roman, 14 points.

Pour les citations : Times New Roman 12 points.

Pour les notes de bas de page : Times New Roman, 12 points.

Édition électronique réalisée avec le traitement de textes

Microsoft Word 2004 pour Macintosh.

Mise en page sur papier format

LETTRE (US letter), 8.5', x 11'’)

Édition complétée le 14 novembre 2005 à Chicoutimi, Ville de Saguenay, province de Québec. 


\section{Bernard Arcand \\ Anthropologue, professeur au département d'anthropologie, Université Laval \\ "Production, culture et idéologie: approche structuraliste."}

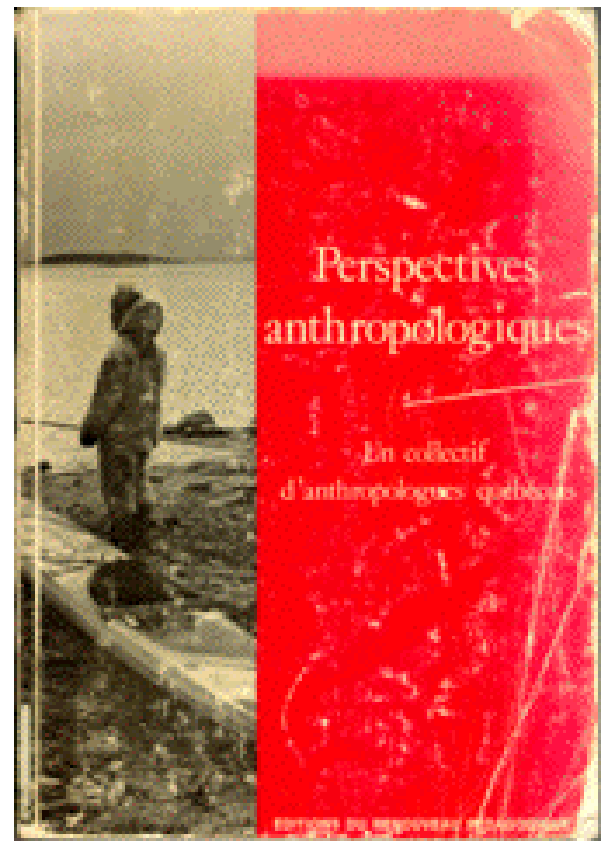

Un article publié dans l'ouvrage collectif intitulé: Perspectives anthropologiques. Un collectif d'anthropologues québécois, chapitre 10, pp. 143 à 153. Montréal: Les Éditions du Renouveau pédagogique, 1979, 436 pp. 


\section{Table des matières}

$\underline{\text { Introduction }}$

1. Tour d'horizon critique des principales théories anthropologiques

2. Recherche d'un mode d'analyse

3. Proposition d'une approche de la société appropriée au changement 
Bernard Arcand

Université Laval

"Production, culture et idéologie : approche structuraliste"

Un article publié dans l'ouvrage collectif intitulé : Perspectives anthropologiques. Un collectif d'anthropologues québécois, chapitre 10, pp. 143-153. Montréal : Les Éditions du Renouveau pédagogique, 1979, 436 pp.

\section{Introduction}

$\underline{\text { Retour à la table des matières }}$

Ce qu'on appelle communément la « culture » consiste en fait en un fouillis, un amas plus ou moins chaotique de tous les comportements humains. Pour gouverner notre société, le pouvoir établi ordonne cette réalité complexe, la découpe en domaines ou catégories. Il distingue ainsi les secteurs de l'économie, des arts, des affaires sociales, des sports et loisirs, etc. Ce mode d'approche de la réalité est conforme à une vieille tradition occidentale. L'anthropologie a longtemps suivi cette tradition et divisé sensiblement de la même manière le monde qu'elle voulait étudier. Pour s'en rendre compte, il suffit de consulter la table des matières de la plupart des monographies dites " classiques », dont les différents chapitres portent sur des sujets tels la culture matérielle, la technologie, l'économie, l'organisation sociale, la politique, la religion, la cosmologie, et aussi la mythologie, les arts et le folklore. L'enseignement de l'anthropologie procède généralement de cette façon et on offre donc aux étudiants des cours en an- 
thropologie économique, sociale, religieuse, etc. Les anthropologues professionnels eux-mêmes se reconnaissent une spécialité dans l'un ou l'autre de ces domaines ; et nous avons ainsi nos ministres de l'anthropologie économique, nos hauts fonctionnaires de la parenté, sans oublier, bien sûr, nos évêques de l'anthropologie de la religion.

Après avoir organisé le vécu en compartiments bien définis afin d'en simplifier la compréhension, le jeu de l'anthropologie a longtemps consisté à découvrir les relations possibles entre ces différents domaines de la réalité sociale. Par exemple, on a essayé de démontrer l'influence de la technologie sur l'organisation sociale, l'importance de la parenté pour la vie économique d'une société, ou les effets déterminants de l'idéologie religieuse sur l'économie. On a ainsi, au cours de l'histoire de la théorie anthropologique, exploré et suggéré tout un éventail de relations possibles, même les plus absurdes, entre les divers aspects de la réalité. Certains demeurent prudents et se limitent à prouver l'existence d'une corrélation statistique entre deux domaines. D'autres, plus hardis, tâchent de convaincre leur auditoire qu'on est en présence d'une relation causale ; ils prétendent que les activités économiques déterminent le type d'organisation sociale, alors que d'autres mettent en relief l'effet déterminant de l'adaptation au milieu physique sur les autres éléments de la culture.

\section{Tour d'horizon critique des principales théories anthropologiques}

$\underline{\text { Retour à la table des matières }}$

Tout va très bien, les anthropologues ont beaucoup de travail et peu de chance d'être contredits. La réfutation de leurs énoncés apparaîtra particulièrement difficile s'ils adoptent l'approche fonctionnaliste, probablement la théorie la plus confortable de toute l'histoire de l'anthropologie. Empruntée à la physiologie sans avoir toujours été bien comprise, la notion de fonction a connu un succès important en anthropologie. En bref, la théorie fonctionnaliste considère la culture 
comme un tout indivisible dont chacune des composantes identifiables est reliée à toutes les autres (chacune influe sur toutes les autres et subit à son tour leur influence, et tout ce jeu d'influences réciproques s'exerce en général de façon positive). Cette théorie mène le plus souvent à une description pure et simple, et n'explique donc rien. On dira, par exemple, que la fonction d'un arc est de chasser, que celle de la chasse est de produire de la nourriture, laquelle sert à nourrir, etc. On explique les choses par leurs fonctions, et ces fonctions réfèrent généralement à la nature même des choses. Cela ne nous aide en rien à comprendre pourquoi une chose existe. De la même manière, vouloir expliquer l'oeil en disant qu'il sert à voir ne ferait que redire ce que l'on savait déjà et précisément ce que l'on cherche à mieux comprendre.

Une autre théorie anthropologique, peut-être encore plus populaire, se désigne sous le nom d'écologie culturelle ou de matérialisme culturel. Alors que les fonctionnalistes recherchent la fonction d'un fait social dans ses relations avec les autres faits sociaux, les écologistes culturels voient cette fonction dans les relations entre les faits sociaux et le milieu naturel environnant. On rencontre tout de suite la même difficulté : les résultats de l'analyse ne font que réintroduire les données du départ et on n'est guère plus avancé. On considère ainsi tout fait social comme partie intégrante du processus d'adaptation au milieu et, après avoir cerné ce fait social à l'aide d'une description souvent très rigoureuse et d'une batterie de statistiques fort impressionnantes, on l'« explique » en précisant qu'il sert à l'adaptation. L'écologie culturelle pourrait, par exemple, expliquer l'existence du service gouvernemental d'assurance-chômage par sa fonction, qui consiste à aider à la survie du groupe au moyen de la redistribution de la richesse collective parmi les membres défavorisés de notre société. On a nettement l'impression que cette conception nous ramène à une description à peine sophistiquée de ce que tout le monde sait, et qui ne prouve rien.

Par contre, le marxisme ou matérialisme historique constitue une approche anthropologique beaucoup plus importante. On peut probablement attribuer son manque de popularité en Amérique du Nord à l'anticommunisme fanatique de nos gouvernements. L'hypothèse fondamentale du matérialisme historique énonce l'existence d'une relation 
déterminante entre les rapports de production et tous les autres aspects de la vie sociale. Directement ou indirectement, les rapports de production exercent une influence déterminante sur toutes les autres catégories, domaines, ou niveaux de la culture. Il suffit donc d'étudier toute société par le biais des rapports de production qui y existent, pour en saisir l'essentiel. De plus, en raison de la cohérence de cette théorie, la seule connaissance de ces rapports de production suffira pour prédire les grandes lignes de tous les autres niveaux de la vie sociale.

Bien sûr, le matérialisme historique ne correspond pas à la perfection et son analyse ne réussit pas à expliquer tous les comportements humains. En effet, certains aspects de la vie sociale révèlent des liens plutôt éloignés avec les rapports de production. Il serait ridicule, par exemple, de prétendre que l'humour est déterminé par la nature des rapports de production; quoique plusieurs manifestations d'humour soient intimement liées au contexte social qui les produit, cela n'explique pas pourquoi des gens de diverses origines sociales et culturelles trouvent drôle le fait de glisser sur une peau de banane. Il serait inapproprié de vouloir englober dans l'analyse marxiste tous les aspects de l'esthétique comme il serait absurde de prétendre que les gens ont des relations sexuelles dans le seul but de produire des enfants. Mais ces critiques bien partielles s'appliquent encore davantage aux autres théories anthropologiques, dont la plupart ne possèdent ni la cohérence, ni le caractère exhaustif du matérialisme historique.

Cependant, une autre objection, beaucoup plus sérieuse, s'adresse non seulement au marxisme mais à toute l'anthropologie. Le matérialisme historique privilégie les rapports de production, ce qui nous amène à nous interroger sur la nature de ces derniers. Or, ils englobent l'ensemble des relations sociales au sein du processus de production (pris dans le sens large d'activité économique et incluant production, transformation et consommation). Cette notion de rapport de production devient particulièrement utile lorsqu'on analyse la société industrielle. Pour comprendre la société québécoise, il est sans doute essentiel d'identifier clairement les rapports de production qui groupent les individus et les font travailler, consommer, et même penser de concert. Mais tout cela se complique terriblement dès qu'on aborde un autre type de société. Par exemple, au sein des sociétés de chasseurs- 
cueilleurs, un homme pourra choisir pour compagnon de chasse son gendre, se placer à l'avant du canot car c'est la position d'un aîné, ou produire de la viande pour l'offrir à son gendre dans le cadre de l'échange viande-légumes qui accompagne tout mariage ; il se servira d'un arc et de flèches, qui sont à la fois outils de production et symboles phalliques; de même, il s'abstiendra de chasser certains animaux qui ne sont pas considérés comestibles, refusera d'exploiter certaines parties du territoire sous prétexte qu'elles sont habitées par des monstres imaginaires, évitera de chasser lorsqu'il participe au rite d'initiation d'une jeune fille qui devient pubère. On pourrait évoquer une foule d'autres détails pour démontrer la complexité de la chasse. Ce qui très vite inquiète l'observateur, c'est la prise de conscience que les fondements mêmes de l'économie ne peuvent être pris pour acquis au début de l'enquête.

Les matières premières, les outils de travail et la main-d'œuvre sont des définitions culturelles. La chasse se base sur un système complexe d'idées comprenant les concepts de nourriture, de canot, de viande, de légumes, de rivière, de savane, de chasse, de cueillette, etc., tous dotés de significations qui dépassent largement ce qu'on entend traditionnellement par " économie ». Si cela exprimait le seul fait qu'on peut constamment conférer aux objets économiques plusieurs autres sens, le problème serait vite résolu. Mais on en vient plutôt à constater que la définition même des choses ne peut se réduire à leur fonction économique. En outre, on ne sait même plus ce qu'est l'économie ou la production. On se rend compte que l'« économie » comporte une bonne part d'« organisation sociale », que cette dernière fait partie de la « religion » qui, elle, est indissociable de la « culture matérielle » et ainsi de suite. On ne peut véritablement procéder au découpage du phénomène global de la chasse en aspects " économique ", " social », et " idéologique ». On se retrouve donc au point de départ, toujours confronté au fouillis de la réalité sociale.

Évidemment, certains anthropologues continueront de manier leurs divisions commodes du réel et de parler des relations entre l'« économie », l'« organisation sociale » et l'« idéologie ». Face à l'importance que prend dans notre société le phénomène de monopolisation des fonctions économiques de la production par un groupe restreint d'individus mus par leurs propres intérêts, les matérialistes historiques 
concluront que l'économie joue toujours un rôle déterminant. D'autres prétendront plutôt que, dans les sociétés de chasseurs-cueilleurs, le système de parenté détermine, envahit tous les domaines de l'activité sociale. Tous réussiront sans doute dans une certaine mesure à démontrer l'existence de liens entre l'« économie », l'« organisation sociale » et l'« idéologie ». Ce succès est prévisible puisque les mêmes personnes produisent des biens et vivent en société, et que, au moment où elles accomplissent ces diverses activités, elles ne tiennent pas compte de ces distinctions anthropologiques. En fait, le succès apparaît évident dès le début, lors de la division arbitraire du réel qui postule comme étant différent ce qui ne l'est pas vraiment ; il est ensuite facile de démontrer des similitudes entre tout cela. Tant que l'on ne pourra établir clairement de quelle manière l'« économie », l'« organisation sociale » et l'« idéologie » diffèrent, il est inutile de les comparer et d'y chercher un déterminisme. On a vu que cette différence entre ces éléments ne semblait pas du tout évidente dès qu'on s'éloignait un peu de notre propre société. Continuer à voir le réel à partir de ces divisions simplistes aurait donc pour effet de dénaturer nos observations ; de plus, ce serait redire ce qui était déjà connu, puisque les conclusions sont contenues dans les prémisses de l'analyse.

\section{Recherche d'un mode d'analyse}

$\underline{\text { Retour à la table des matières }}$

Il nous faut donc repartir à zéro et ignorer la majorité des travaux anthropologiques disponibles jusqu'à maintenant. Ainsi, si nous faisons abstraction de notre intérêt pour l'histoire de l'anthropologie, il semble que l'anthropologie ait jusqu'à présent dit peu de choses vraiment valables à propos des sociétés non occidentales qu'elle a étudiées, et encore moins sur la nature humaine. L'anthropologie nous en a toujours dit beaucoup plus sur les anthropologues que sur les autres humains. Soulignons cependant que l'anthropologie peut tout de 
même compter sur quelques acquis puisque, alors que la plupart des anthropologues s'enlisaient dans des querelles tautologiques, d'autres, particulièrement certains linguistes, psychologues, philosophes et ingénieurs de la communication, établissaient les bases d'une nouvelle anthropologie.

Les psychologues spécialistes du développement de l'enfant nous ont appris que l'être humain pense à l'aide de catégories. C'est-à-dire que tout objet matériel, toute personne, tout comportement, toute idée sont groupés à l'intérieur d'une classe d'objets que, d'une part, nous définissons comme semblables entre eux, et, d'autre part, comme différents des objets appartenant aux autres classes. Par exemple, je conçois la chaise qui est devant moi comme faisant partie de la catégorie chaise qui inclut tous les meubles comprenant quatre pattes, un dossier, un siège, etc., et comme étant différente des objets qui entrent dans les catégories banc, tabouret, fauteuil, etc. Or, fait crucial pour l'anthropologie, mon comportement face à cette chaise est en grande partie déterminé par mon comportement face à tout objet de la catégorie chaise : "les chaises sont faites pour s'asseoir ».

L'esprit humain a tendance à former ce genre de catégories, dans le but de mettre de l'ordre dans le chaos qui l'entoure. Par exemple, dans la nature, le spectre des couleurs se présente à nous sous l'aspect d'un continuum qui va de l'ultra-violet à l'infrarouge, et qui n'admet pas de distinctions internes. Pourtant, nous inventons des divisions, nous formons des catégories indépendantes et exclusives que nous appelons le bleu, le vert, le rouge, le jaune, etc. Nous créons artificiellement des séparations dans ce continuum et nous en venons à concevoir les couleurs comme des unités distinctes et autonomes qui existent vraiment dans la nature. Toutefois, ce découpage de la nature ne s'opère pas toujours pareillement et les divers groupes humains organisent leur perception de la nature selon des schèmes différents. Pour reprendre l'exemple des couleurs, plusieurs groupes d'Indiens du Brésil n'établissent pas comme nous de discrimination entre le bleu et le vert (plusieurs langues indiennes utilisent le même terme pour décrire ces deux couleurs), alors que cette distinction importe pour nous (nos critères esthétiques nous ont appris à dissocier ces deux couleurs qui « ne vont pas ensemble »). 
Or, il en est de même pour tout ce qui nous entoure. Des gens différents (de « culture » différente, mais souvent aussi au sein de ce que l'anthropologie traditionnelle appelle la même " culture ») organisent le monde de manière différente et donnent ainsi des sens différents aux choses, aux personnes et aux idées qui les entourent. L'examen des catégories que nous établissons à propos de nos parents révèle que nous ne distinguons pas entre les cousins parallèles (les enfants du frère du père et de la sœur de la mère) et les cousins croisés (les enfants de la sœur du père et du frère de la mère) : tous ces gens constituent pour nous des "cousins ». Par contre, dans d'autres sociétés, cette distinction est tout à fait essentielle, puisque les cousins parallèles sont appelés " frères et sœurs ", tandis que les cousins croisés sont ceux que l'on doit épouser, et qui ne sont pas considérés comme des frères et des sœurs. Dans notre société, le choix de ces termes marque l'importance de la démarcation entre la famille nucléaire et le reste de la société. Ailleurs, on distingue nettement entre cousin parallèle et cousin croisé, entre personne de même sexe et personne de sexe opposé. Il nous faut découvrir ces critères propres à chaque société, puisqu'ils constituent le fondement même des catégories et que ces dernières, qui ont pour but de mettre de l'ordre dans le monde et de donner un sens à toute chose, forment donc la base de toute vie sociale. Si l'on s'obstine dans des comparaisons superficielles telles que l'utilisation des couleurs chez les Indiens du Brésil et chez nous, ou les systèmes de parenté dans leurs manifestations morphologiques (système " iroquois » par rapport au système « hawaïen », etc.), on persiste à ne jamais comparer que des poires et des oranges. Il ne semble pas inutile de réitérer l'affirmation selon laquelle ce travail de catégorisation est nécessaire à toute vie humaine puisqu'il permet de fonder un ordre des choses et de donner à la vie un minimum de sens. Si nous n'avions assimilé le concept assez précis de chaise, chaque fois que nous nous trouvons face à l'objet correspondant à ce concept, ce serait pour nous une expérience entièrement nouvelle que nous aurions sans doute de la difficulté à comprendre. Il est impossible d'imaginer la vie sociale comme une suite d'expériences uniques, et tellement plus simple de s'approcher d'une chaise, de s'asseoir et de pouvoir consacrer son temps à des choses plus importantes. D'autre part, cette conception des choses doit être partagée par les membres d'une même communauté humaine, qui agissent les uns sur les autres. Si un tel partage n'existait pas, toute communication et, par conséquent, toute forme d'échange et 
toute vie sociale deviendraient impossibles. Dans ce contexte, l'exemple du langage demeure peut-être le plus adéquat. Si nous avons quelque chose à dire à quelqu'un, il faut que nous nous exprimions selon certaines règles, sinon nous ne serons pas compris. La phrase que nous voulons formuler doit obéir aux lois de la grammaire, sinon le message ne pourra être capté par l'autre ; par exemple : "Est pense ronde je terre la que », est une phrase absurde et inutile, car elle a pour effet d'abolir la communication. L'existence d'un code utilisé par plusieurs individus et le respect des mêmes lois de la grammaire par les interlocuteurs rendent possibles la conversation et l'échange d'information entre eux. Depuis longtemps, on a admis l'importance des lois grammaticales précises dans la communication verbale. Mais les anthropologues ont plus de difficulté à comprendre que la parole, comme type de comportement social, ne diffère pas essentiellement de tout autre type de comportement. Pour avoir un sens et être compris par d'autres, tout geste, toute action sociale doit obéir aux règles d'un code commun à tous. Toute action prend un sens social en référence à ce code. Ainsi, l'acte de tourner en rond sur soi-même le plus vite possible est réservé dans notre société à certaines catégories de gens qui peuvent l'accomplir en des circonstances assez précises : les danseurs de ballet, les patineurs artistiques, les enfants surexcités et les aliénés. $\mathrm{Si}$, un jour, au cours d'une conférence de presse, le premier ministre entreprenait de tourner sur lui-même à grande vitesse, l'assistance, après avoir examiné et rejeté diverses interprétations possibles de ce fait (il n'est plus un enfant, il est trop âgé pour faire du ballet, il ne porte pas de patins, etc.) en viendrait à conclure qu'il est devenu aliéné. C'est là le sens que la société attacherait à son geste, en procédant de manière tout à fait logique à partir des catégories préalablement établies et des relations qui existent entre ces catégories tourner en rond, premier ministre, conférence de presse, danseur, patineur, enfant, folie, etc. Il est bien évident, d'autre part, que l'acte lui-même n'a pas de signification en soi et que plusieurs façons de le catégoriser existent ; ainsi, alors que chez nous l'action de tourner en rond est assez rare et inusitée, elle devient ailleurs un élément essentiel d'une performance chamanique ou une forme de danse de réjouissance.

A la suite de cet exemple qui pourrait paraître simpliste, une distinction tout à fait cruciale s'impose. Ces règles de comportement humain, ces lois de la logique du code ne correspondent pas à ce qu'on 
appelle généralement les valeurs, la morale ou l'idéologie d'une société. Le système des valeurs se définit plutôt comme un ensemble de préceptes moraux dont les gens sont plus ou moins conscients, mais qu'ils utilisent pour guider leur vie, ou contre lesquels ils se révoltent. Au contraire, les règles dont il est question ici ont le plus souvent un caractère inconscient, c'est-à-dire que nous leur obéissons sans nous en rendre compte. Ainsi, lorsque nous parlons français, nous respectons les règles de la grammaire française, souvent sans le savoir. Nous avons tous appris les rudiments de la grammaire française (que nous avons vite oubliés), mais chaque fois que nous nous exprimons, il n'est pas nécessaire de revoir ces règles; nous les utilisons inconsciemment. Et les linguistes ont suffisamment poussé leur analyse des règles du langage pour pouvoir prédire avec précision, non pas ce que nous pouvons dire, mais comment nous pouvons le dire. Nous connaissons maintenant les limites du langage.

La distinction entre conscient et inconscient est souvent très difficile à établir puisqu'elle se fonde sur la croyance selon laquelle on peut saisir véritablement les mécanismes de la pensée (ce à quoi peu d'anthropologues prétendent). Nous l'introduisons ici tout d'abord pour mettre en évidence ce qui souvent distingue ces règles de ce qu'on appelle les valeurs, mais aussi parce que pour certains, qui retombent par là dans l'habitude stérile de la catégorisation propre à l'anthropologie, ces règles de la grammaire du comportement humain font nécessairement partie de l'idéologie, c'est-à-dire qu'elles sont plus ou moins consciemment véhiculées par les gens et correspondent à une sorte de rationalisation postérieure au comportement. En fait, le caractère conscient ou inconscient de ces règles n'importe aucunement. on devrait plutôt s'intéresser à la façon dont elles fondent et permettent le comportement. Il importe également de voir comment les valeurs et l'idéologie reposent elles-mêmes sur un ordre logique. Ainsi, affirmer que, dans notre société, l'idéologie dominante impose le respect de l'autorité revient à dire que les individus qui appuient et propagent cette idéologie, comme ceux qui s'y opposent et veulent la détruire, partagent un concept d'autorité, découlant en partie de la notion d'inégalité, elle-même définie par son opposition au concept d'égalité. Le tout forme un ensemble logique sans lequel il n'y aurait ni opium du peuple, ni révolution. 
L'anthropologie doit donc utiliser une démarche semblable àcelle de la linguistique. Dans le désordre des phrases, le linguiste cherche certaines constantes, desquelles découlent ensuite des lois permettant de comprendre comment toutes ces phrases, pourtant si différentes, se ressemblent toutes en ce qu'elles obéissent à ces mêmes règles. Il peut ainsi prédire non seulement comment sera notre phrase, mais aussi comment seraient celles que nous ne dirons jamais. Jusqu'à maintenant, les anthropologues se sont trop souvent limités à la comparaison pure et simple des phrases entre elles, des comportements entre eux, comme si on pouvait tirer quelque chose à joindre une phrase chinoise et une phrase française. Pour aller plus loin, il faudra analyser chaque comportement en lui-même et en dégager la logique interne, afin d'en établir la grammaire. C'est seulement ensuite que l'on pourra vraiment faire des comparaisons et juger de leur utilité. Autrement, on demeure en quelque sorte « trop près des arbres pour percevoir la forêt ».

Avant d'imposer au monde les concepts occidentaux de " production » et "d'idéologie », il faut savoir de quoi nous parlons. Cela requiert, dans chaque société, une analyse rigoureuse de chacun de ces domaines artificiels afin d'en dégager la cohérence interne et les lois qui gouvernent la logique des deux types d'activité. Alors, et dans ce seul contexte, serons-nous en position d'affirmer, d'abord, que ces deux domaines existent vraiment et qu'ils sont autonomes, ensuite, qu'une certaine relation existe entre eux. On pourra ensuite parler, sans ethnocentrisme, de production, d'organisation sociale et d'idéologie.

En fait, lorsqu'on adopte une telle approche, ses résultats nous éloignent des divisions traditionnelles de l'anthropologie. Si on s'intéresse à un "domaine » de la réalité sociale tel la "production », on recherchera avant tout les lois qui régissent les catégories de matières premières, les catégories d'outils, les catégories de groupes de production, les catégories d'effets de l'action humaine sur la nature, etc. Face à un outil, on verra quels critères le différencient des autres outils (ce qui fait, par exemple, qu'un marteau n'est pas une masse ou une hache). On découvrira aussi comment ces critères qui ordonnent tous les outils forment nécessairement un ensemble logique ; si ce n'était pas le cas, il y aurait confusion et on ne pourrait reconnaître un outil d'un autre, et encore moins l'utiliser; si l'outil ne s'intégrait pas dans un 
système logique d'idées, il n'existerait tout simplement pas, puisqu'il n'aurait aucun sens. Lorsqu'on aura saisi la cohérence de l'ordre qui règne parmi les outils, les matières premières, les concepts d'action sur la nature, etc., on dira que la logique de la production réside tout simplement dans l'ensemble des principes logiques qui fondent toutes ces lois. Ces lois, qui pourtant régissent des réalités apparemment très différentes, en viennent à se présenter à nous comme autant de manifestations particulières d'une même logique : le système cohérent qui organise les outils et permet leur compréhension, donc leur fabrication et leur utilisation, sera identique à celui qui régit les matières premières, les groupes de production, etc. Or, lorsqu'on pousse plus loin l'analyse et qu'on étudie non seulement la logique de la production, mais aussi celle de l'idéologie ou de tout autre « domaine » social, on arrive à voir qu'à un niveau suffisant d'abstraction, ces logiques se ressemblent toutes. C'est à ce moment que les «domaines »disparaissent comme entités indépendantes, Puisqu'ils se limitent à être les manifestations particulières d'un nombre limité et constant de principes logiques. La « production » rejoint alors « l'idéologie » et il devient inutile de les comparer ou de les mettre en relation.

Dans ce contexte, on peut soulever de nouveau le problème de la comparaison entre des groupes sociaux distincts. Mais, au lieu de comparer les cultures au niveau le plus superficiel, on établira la comparaison entre les différents systèmes logiques. Plutôt que d'opposer leurs manifestations particulières (qui varient considérablement en différentes parties de la terre et en différents moments de l'histoire), on comparera les lois qui régissent ces manifestations, la logique qui sous-tend ces réalités concrètes et directement observables.

On soulèvera ensuite les questions essentielles : cette logique de la pensée humaine est-elle universelle, et d'où provient-elle ? Il est encore trop tôt pour répondre à ces questions, mais on peut dès maintenant imaginer (ce que certains, en fait, postulent) que la composition biochimique du cerveau humain lui impose certaines formes de codification, certains modes de pensée. En ce sens, tous nos comportements, toute notre vie sociale seraient en premier lieu déterminés par la nature (matérielle) de notre cerveau. Ces questions intéressent depuis longtemps la physiologie comme la philosophie et il est peu probable qu'une réponse définitive vienne des anthropologues. Notre rôle 
consisterait plutôt à continuer de démontrer que les êtres humains sont partout semblables au point de vue intellectuel, que tout élément culturel est le produit de leur cerveau et qu'en recherchant la logique interne de ces éléments culturels, on contribue à la connaissance de la composition du cerveau humain. Ce serait là sans doute la première constatation intéressante que l'anthropologie aurait faite sur la nature humaine.

\section{Proposition d'une approche de la société appropriée au changement}

$\underline{\text { Retour à la table des matières }}$

Entre-temps, il nous faut aussi aborder un autre point important. Ce genre d'analyse formelle ne mène-t-il pas à la découverte de structures logiques qui, sans nier leur valeur, sont néanmoins tout à fait statiques et immuables? On escamoterait ainsi complètement le changement, un des aspects majeurs de toute vie sociale. En guise de réponse, mentionnons que l'anthropologie cherche à comprendre la nature humaine ; elle s'intéresse donc surtout, par définition, à ce qui est permanent. Ainsi, on privilégie l'étude du changement social dans le but de dégager la permanence des conditions de son apparition.

D'autre part, il y a plusieurs raisons de croire que l'approche suggérée ici constitue celle qui permet le mieux de saisir le changement. Reprenons, une dernière fois, l'exemple de la chaise. Une chaise nous apparaît comme un objet original et unique jusqu'au moment où, rencontrant une deuxième chaise, nous voyons que les deux objets possèdent en commun certains critères particuliers qui servent à définir toute chaise. Nous penserons alors au concept de chaise qu'aucun des deux objets ne représente de façon exhaustive : il nous sera alors évident qu'il n'y a pas que ces deux chaises dans le monde et que plusieurs autres chaises peuvent exister; il nous sera même possible de créer de nouvelles chaises. Mieux nous connaîtrons la logique for- 
melle du meuble chaise, plus nous pourrons prévoir plusieurs autres chaises possibles, même si elles n'ont pourtant jamais été fabriquées et si aucun anthropologue n'a encore pu les observer.

Nous aboutissons alors à la notion de "programme », telle qu'élaborée par ceux qui utilisent des ordinateurs. Il faut comprendre le " programme » intitulé chaise pour être en mesure de prévoir toutes les chaises possibles; de même, il nous faut atteindre les "programmes " intitulés cultures pour pouvoir comprendre leurs transformations. Le réel, tel qu'on l'observe, est une manifestation particulière d'un "programme » qui inclut plusieurs autres manifestations possibles. Il est donc nécessairement plus facile de prévoir le changement lorsque nous sommes conscients, non seulement de ce qui se passe vraiment, mais aussi de tout ce qui est possible. Sur ce point, les sciences naturelles jouissent d'un avantage marqué sur l'anthropologie. Le chimiste peut vérifier le " programme » qu'il a élaboré en créant dans son laboratoire, expérimentalement, toutes les transformations de sa théorie. Tandis que nous, pour vérifier nos "programmes », devons attendre que les sociétés se transforment. Mais l'essentiel demeure quand même de travailler à l'élaboration de tels "programmes ", car la seule observation du réel nous laissera toujours totalement dépourvus face au changement, précisément parce qu'il est nouveau et parce qu'il nous confronte avec de l'inédit.

Enfin, après avoir mené à terme l'analyse du comportement, des institutions, ou des «domaines » privilégiés de la vie sociale, et identifié leur logique formelle pour en élaborer le "programme », nous répondrons aux questions que plusieurs voient comme dominantes aujourd'hui en anthropologie, mais qu'un trop grand nombre aborde de manière beaucoup trop hâtive. Si, comme nous l'avons soutenu, le réel observé n'est qu'une des manifestations possibles d'un " programme » connu, pourquoi rencontrons-nous cette manifestation bien particulière au sein d'une société définie et à un moment historique bien précis ? Dire qu'une chaise dessinée par le danois Arne Jacobsen constitue un autre exemple de la logique occidentale du concept de chaise n'explique pas pourquoi ses lignes sont aussi fines, pourquoi elle fut créée au Danemark, ni pourquoi on ne peut l'acheter au sous-sol de tel magasin. Toutes ces questions doivent trouver réponse, et c'est alors que nous faisons appel à certaines données de l'anthropologie tradi- 
tionnelle (puisqu'on retourne alors au niveau du réel observable). En particulier, les contributions du matérialisme historique nous aideront sans doute à définir les conditions selon lesquelles le "programme » se transforme.

Cependant, il est tout à fait essentiel d'adopter la bonne méthodologie et de mener avant tout l'analyse formelle des faits sociaux, d'en dégager la structure logique et d'en élaborer le « programme ». Procéder à l'inverse et introduire dès le début de l'enquête les notions de base du matérialisme historique nous replongerait dans l'erreur de l'anthropologie traditionnelle. On imposerait au réel un ordre qui correspond davantage à l'état d'esprit de notre société qu'à la nature humaine. On parierait de rareté de biens sans se rendre compte que ce concept n'a de sens que si l'on prend pour acquis le concept de maximisation. On parlerait de "travail », sans savoir à quoi cela correspond chez ceux dont on parle. On prendrait comme point de départ une certaine "volonté de survivre ", sans jamais expliquer que, pour vouloir vivre, il faut que la vie ait un sens, que la production soit rationnelle, et que cette volonté de survivre est elle-même un produit.

Dans notre tentative de choisir une approche qui cerne la réalité, il est facile de tomber dans l'erreur que nous avons signalée, d'autant plus que le matérialisme historique constitue sans doute l'approche anthropologique qui nous a le plus appris sur notre société et qui guide le mieux notre action politique. Toutefois, l'adoption de l'approche mise de l'avant par le matérialisme historique comporte autant de dangers que celle des approches de l'anthropologie traditionnelle : le matérialisme historique mène surtout à l'étude d'une toute petite culture, la culture de l'anthropologie ; d'autre part, il devient une forme de cannibalisme intellectuel et perpétue ainsi le rôle de l'anthropologie comme forme directe et brutale d'agression et s'oriente dans le même sens que la destruction du monde par l'Occident.

Enfin, un autre risque nous guette, celui qui consiste en une première étape à accepter la critique formulée ici, mais à ajouter que tout cela a finalement bien peu d'importance puisque « ce qui nous intéresse, c'est notre société, c'est le Québec ». Prétendre que le matérialisme historique demeure pleinement valide puisqu'il nous permet de mieux comprendre le Québec, même s'il a tort lorsqu'il cherche à ex- 
pliquer les sociétés de chasseurs-cueilleurs, c'est affirmer qu'une erreur au sujet des "primitifs » est moins grave qu'une erreur sur notre société. À ce moment, l'anthropologie devient raciste. 